0049-3848(94)E0013-3

\title{
A METIIOD FOR THE QUANTITATION OF PROTAMINE IN PLASMA
}

\author{
Victor C. Yang1, You-Yin Fu' ${ }^{1}$, Ching-Leou C. Teng1, Shu-Ching Ma1,2, \\ and Jacob N. Shanberge 3 \\ ${ }^{1}$ College of Pharmacy, The University of Michigan, Ann Arbor, MI 48109-1065, 2 Nova \\ Biomedical Corp., 200 Prospect Street, Waltham, MA 02254, and ${ }^{3}$ Hemostasis and Coagulation \\ Laboratory, William Beaumont Hospital, Royal Oak, MI 48072, USA
}

(Received 5 October 1993 by Editor B. Dahlbäck; revised/accepted 2 March 1994)

Abstract A unique and simple colorimetric method for the quantitation of plasma protamine levels has been developed. The method is established on the competitive binding displacement mechanism between protamine and heparin-azure A dye complex, and the metachromatic color change of azure A dye in the presence of heparin. Because the method is based on the clinical specificity of protamine as the heparin antagonist, it is specific for protamine quantitation. Plasma protamine levels determined by this method are within $94 \%$ of accuracy when compared with their aqueous counterparts determined by the conventional Lowry protein assay. Since the method measures the protamine excess after heparin neutralization, it potentially could be employed during clinical heparin reversal with protamine to monitor protamine excess. In addition, the method may provide a useful means to identify the mechanism of the so-called "heparin rebound".

Protamine is a low molecular weight protein rich in lysine, arginine and other basic amino acids, and is generally obtained from fish sperm of the Salmonidae family (1). It is administered regularly following cardiopulmonary bypass and cardiac catheterization to reverse the anticoagulant activity of heparin $(2,3)$. The basic amino groups of protamine complex electrostatically with the sulfate groups of heparin, rendering heparin's anticoagulant effect ineffective. Due to the lack of aromatic amino acid residues such as tyrosine, phenylalanine and tryptophan, protamine, unlike most protein or peptide molecules, does not absorb light in the ultraviolet region (i.e. $280 \mathrm{~nm}$ ). Although protamine interacts with the Folin-phenol reagent and Coomassie Brilliant Blue G-250 and thus can be measured with either the Lowry (4) or Bradford method (5), these protein assays are not specific for protamine. In addition, protamine is devoid of any catalytic and enzymatic activities. All of these factors render the quantity of protamine less assessable, particularly in a complex medium such as plasma where a variety of proteins are present.

Presented herein is a unique method for the accurate quantitation of protamine in plasma samples. The method is established on the competitive binding displacement mechanism between protamine, heparin and azure A dye. Azure A dye is known to bind to heparin, and the interaction results in a

Key words: Protamine quantitation; protamine excess; azure A dye; metachromasia; heparin. Corresponding author: Dr. Victor C. Yang, Associate Professor, College of Pharmacy, The University of Michigan, Ann Arbor, Michigan 48109-1065. 
metachromatic shift of the dye's absorption maximum from 620 (blue) to $530 \mathrm{~nm}$ (purple) (6). The binding is, however, reversible and relatively weak compared to that between heparin and protamine. Thus when protamine is present, it will bind to heparin and displace a certain portion of the dye from the heparin-azure A complex. Once the dye is dissociated from heparin, its absorption maximum immediately shifts back to the initial wavelength of $620 \mathrm{~nm}$. The degree of dye displacement is linearly proportional to the protamine concentration in the sample. Therefore, by adding the test plasma sample to an assay mixture containing heparin-azure $A$ dye complexes and then measuring the absorbance at $620 \mathrm{~nm}$, the protamine content in the sample can be precisely determined from a pre-constructed standard curve.

Determined in this study is the sensitivity and accuracy of the method for protamine quantitation, as well as the effect of sample variation on the slope of the standard curve. In addition, by adding protamine to an already heparinized plasma sample, we here examined whether the method measures the total protamine content or the "protamine excess" after heparin neutralization.

\section{MATERIALS AND METHODS}

Liquid commercial heparin (1000 units/mL) was obtained from Elkins-Sinn (Cherry Hill, NJ). This heparin was from porcine intestinal mucosa, and possessed an average molecular weight of 11,500 daltons and a specific activity of 165 USP units/mg. Protamine sulfate (Clupeine, from Herring) and the Folin-phenol reagent for the Lowry protein assay were purchased from Sigma Chemical Co. (St. Louis, MO). The dye reagent required for the Bradford protein assay was purchased from Bio-Rad Laboratories (Richmond, CA). Azure A dye (A-970, lot 741002) was from Fisher Scientific Co. (Fair Lawn, NJ). Actin (activated cephaloplastin reagent) for the aPTT assay was from Dade (Miami, FL). The aPTT assay was performed using the procedures described previously (7). Freshly frozen citrated (CPD) human plasma was obtained from the American Red Cross (Detroit, $\mathrm{MI}$ ), and was stored at $-40^{\circ} \mathrm{C}$ prior to its use. Water was distilled and de-ionized. Unless otherwise stated, all experiments were conducted at room temperature (20 $\pm 1^{\circ} \mathrm{C}$ ) and each experiment was performed in triplicate.

Construction of Standard Curves.

Assay solutions containing $80 \mu \mathrm{g} / \mathrm{mL}$ of azure A dye and 3.3,5,10,15 units/mL of heparin were prepared by the addition of adequate amounts of azure A dye and heparin to distilled water. These heparin-dye assays solutions, when stored refrigerated, remain stable for a period of at least three months. From each assay solution, aliquots of $600 \mu \mathrm{L}$ were drawn and transferred to a sequence of polypropylene tubes. To each tube, $400 \mu \mathrm{L}$ of plasma containing increasing amounts of protamine were added. The absorbance in each tube was then measured at $620 \mathrm{~nm}$ against the reference sample which contained the same amount of heparin and azure A dye but no protamine. The standard curves thus prepared with final heparin concentrations of $2,3,6$, and 9 units $/ \mathrm{mL}$ were defined as the "2-", "3-", "6-", and "9-unit standard", respectively.

\section{Validation of the Method for Protamine Quantitation.}

Two sets of test samples parallel in protamine concentrations ranging from 25 to $250 \mu \mathrm{g} / \mathrm{mL}$ were prepared from the same protamine stock solution $(6 \mathrm{mg} / \mathrm{mL})$ using adequate dilutions. These two sets of samples were prepared in exactly the same way except that one set utilized water and the other set utilized plasma as the dilution vehicle. Protamine concentrations in aqueous samples were then determined by the Lowry method (4), while protamine concentrations in plasma samples were determined by the dye method. Each concentration measurement was performed in triplicate. To perform the dye method, $400 \mu \mathrm{L}$ of test samples with protamine concentrations in the range of 0$40,40-90,90-175$, and $175-250 \mu \mathrm{g} / \mathrm{mL}$ were added to $600 \mu \mathrm{L}$ assay solutions containing $3.3,5$, 10 , and 15 units $/ \mathrm{mL}$ of heparin, respectively. All the assay solutions contained $80 \mu \mathrm{g} / \mathrm{mL}$ of azure A dye. Protamine concentrations in the above four concentration ranges were then determined using the "2-", "3-", "6-", and "9-unit" standard curves, respectively. Both the test protamine samples and the standard curves were prepared with plasma obtained from the same donor. The two protamine quantitation methods were compared statistically using the method-comparison procedures described by Westgard and Hunt (8). Linear regression analysis was performed to 
metachromatic shift of the dye's absorption maximum from 620 (blue) to $530 \mathrm{~nm}$ (purple) (6). The binding is, however, reversible and relatively weak compared to that between heparin and protamine. Thus when protamine is present, it will bind to heparin and displace a certain portion of the dye from the heparin-azure A complex. Once the dye is dissociated from heparin, its absorption maximum immediately shifts back to the initial wavelength of $620 \mathrm{~nm}$. The degree of dye displacement is linearly proportional to the protamine concentration in the sample. Therefore, by adding the test plasma sample to an assay mixture containing heparin-azure $A$ dye complexes and then measuring the absorbance at $620 \mathrm{~nm}$, the protamine content in the sample can be precisely determined from a pre-constructed standard curve.

Determined in this study is the sensitivity and accuracy of the method for protamine quantitation, as well as the effect of sample variation on the slope of the standard curve. In addition, by adding protamine to an already heparinized plasma sample, we here examined whether the method measures the total protamine content or the "protamine excess" after heparin neutralization.

\section{MATERIALS AND METHODS}

Liquid commercial heparin (1000 units/mL) was obtained from Elkins-Sinn (Cherry Hill, NJ). This heparin was from porcine intestinal mucosa, and possessed an average molecular weight of 11,500 daltons and a specific activity of $165 \mathrm{USP}$ units/mg. Protamine sulfate (Clupeine, from Herring) and the Folin-phenol reagent for the Lowry protein assay were purchased from Sigma Chemical Co. (St. Louis, MO). The dye reagent required for the Bradford protein assay was purchased from Bio-Rad Laboratories (Richmond, CA). Azure A dye (A-970, lot 741002) was from Fisher Scientific Co. (Fair Lawn, NJ). Actin (activated cephaloplastin reagent) for the aPTT assay was from Dade (Miami, FL). The aPTT assay was performed using the procedures described previously (7). Freshly frozen citrated human plasma was obtained from the American Red Cross (Detroit, MI), and was stored at $-40^{\circ} \mathrm{C}$ prior to its use. Water was distilled and deionized. Unless otherwise stated, all experiments were conducted at room temperature $\left(20 \pm 1^{\circ} \mathrm{C}\right)$ and each experiment was performed in triplicate.

\section{Construction of Standard Curves.}

Assay solutions containing $80 \mu \mathrm{g} / \mathrm{mL}$ of azure A dye and 3.3, 5, 10, 15 units/mL of heparin were prepared by the addition of adequate amounts of azure A dye and heparin to distilled water. These heparin-dye assays solutions, when stored refrigerated, remain stable for a period of at least three months. From each assay solution, aliquots of $600 \mu \mathrm{L}$ were drawn and transferred to a sequence of polypropylene tubes. To each tube, $400 \mu \mathrm{L}$ of plasma containing increasing amounts of protamine were added. The absorbance in each tube was then measured at $620 \mathrm{~nm}$ against the reference sample which contained the same amount of heparin and azure A dye but no protamine. The standard curves thus prepared with final heparin concentrations of $2,3,6$, and 9 units $/ \mathrm{mL}$ were defined as the "2-", "3-", "6-", and "9-unit standard", respectively.

\section{Validation of the Method for Protamine Quantitation.}

Two sets of test samples parallel in protamine concentrations ranging from 25 to $250 \mu \mathrm{g} / \mathrm{mL}$ were prepared from the same protamine stock solution $(6 \mathrm{mg} / \mathrm{mL})$ using adequate dilutions. These two sets of samples were prepared in exactly the same way except that one set utilized water and the other set utilized plasma as the dilution vehicle. Protamine concentrations in aqueous samples were then determined by the Lowry method (4), while protamine concentrations in plasma samples were determined by the dye method. Each concentration measurement was performed in triplicate. To perform the dye method, $400 \mu \mathrm{L}$ of test samples with protamine concentrations in the range of 0 $40,40-90,90-175$, and $175-250 \mu \mathrm{g} / \mathrm{mL}$ were added to $600 \mu \mathrm{L}$ assay solutions containing $3.3,5$, 10 , and $15 \mathrm{units} / \mathrm{mL}$ of heparin, respectively. All the assay solutions contained $80 \mu \mathrm{g} / \mathrm{mL}$ of azure A dye. Protamine concentrations in the above four concentration ranges were then determined using the "2-", "3-", "6-", and "9-unit" standard curves, respectively. Both the test protamine samples and the standard curves were prepared with plasma obtained from the same donor. The two protamine quantitation methods were compared statistically using the method-comparison procedures described by Westgard and Hunt (8). Linear regression analysis was performed to 
estimate the accuracy, constant and proportional errors of the method. For linear regressions we used the averaged values from the comparison methods as the independent variable.

\section{The Effect of Sample Variation on the Slope of the Standard Curve.}

The "3-unit standard" was performed on seven plasma samples obtained from the American Red Cross from completely different donors. The slope of each curve was determined and statistically compared.

\section{Determination that the Method Measures Protamine Excess.}

Protamine excess is defined as the excess amount of free protamine that remains in the heparinized sample after all the heparin in the sample is neutralized by protamine. To determine whether the method measures the total protamine added or only the protamine excess, $216 \mu \mathrm{g}$ of protamine were added to $2 \mathrm{~mL}$ of plasma sample pre-treated with 6 units of heparin. Four hundred microlitres of the sample were then added to $600 \mu \mathrm{L}$ of assay solution containing $80 \mu \mathrm{g} / \mathrm{mL}$ of azure A dye and 5 units/mL of heparin, and the protamine content was measured using the "3-unit standard". A standard protamine titration using procedures described in a previous publication (7) was performed on the original heparinized sample to determine the equivalent value of heparin neutralization with protamine. Each experiment was performed in triplicate.

\section{RESULTS}

In Figure 1 are the standard curves constructed with final heparin concentrations of 3,6, and 9 units/mL. Each curve is characterized by a sigmoid shape which contains a well-defined linear region sandwiched by two (a lower and an upper) plateaus. In the lower plateau region, the protamine levels in the samples are too low relative to heparin, and thus insufficient amounts of

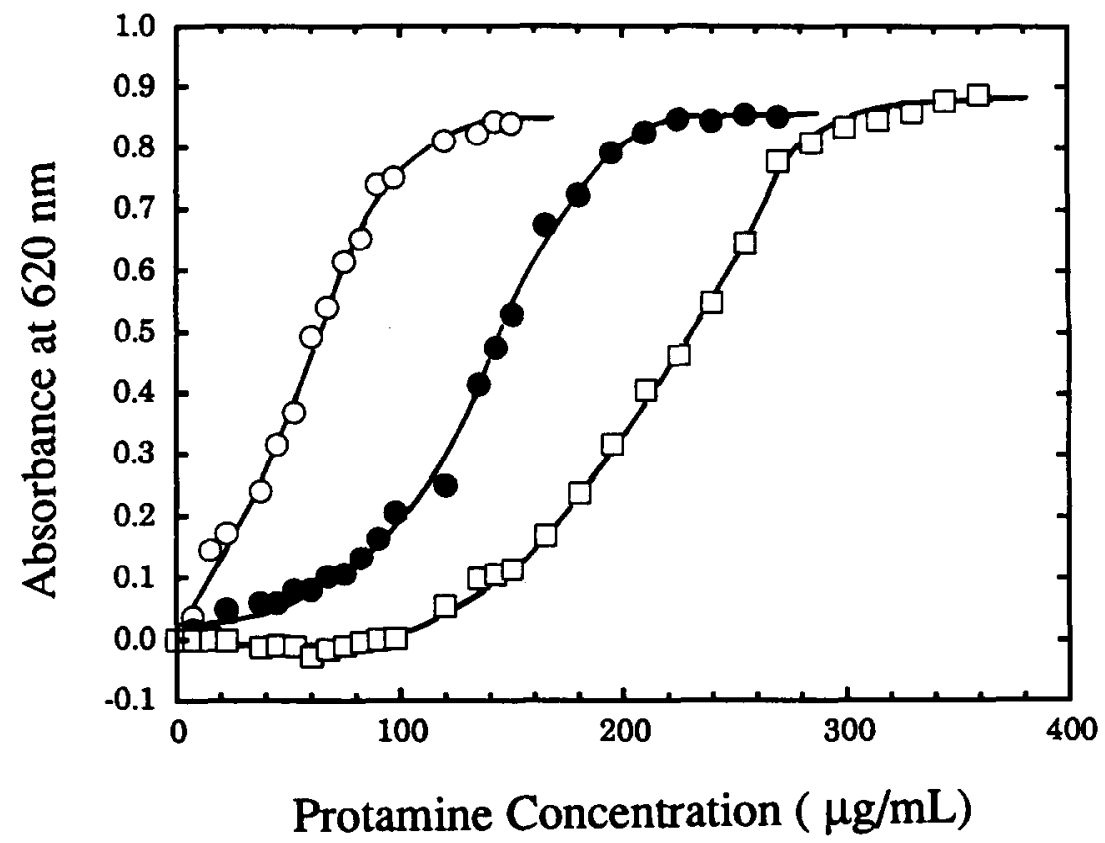

FIG. 1

Representative standard curves of the assay constructed with a final heparin concentration of $(\mathrm{O}-\mathrm{O}), 3$ units $/ \mathrm{mL}$; ( $-\mathrm{O}$ ), 6 units $/ \mathrm{mL}$; and $(\square-\square), 9$ units $/ \mathrm{mL}$. Each experiment was performed in triplicate. The standard deviation of the absorbance at $620 \mathrm{~nm}$ at each protamine concentration was always within \pm 0.01 absorbance unit. To avoid overcrowding, standard deviations of the data points are not included in the figure. 
dye are displaced from the heparin-dye complex. In the upper plateau region, however, protamine is present in large excess so that the maximum amount of dye is replaced from the heparin-dye complex and into the solution. The linear region of the curve represents the assay range of the method. It should be noted that the intercepts of the linear region with the two plateaus reflect the two detection limits (i.e. the lower and upper limits), whereas the slope of the linear region represents the sensitivity of the method. Increasing heparin concentration in the final assay mixture from 3 to 9 units $/ \mathrm{mL}$ results in a shift of the detection limits towards higher protamine concentrations, in conjunction with a concomitant slight reduction of the slope from 0.290 to 0.250 . These results suggest that when samples with higher protamine concentrations are assayed, higher heparin levels in the assay solution may be needed, although this could result in reduction of the sensitivity of the method.

As seen in Figure 2, the method compares favorably with the conventional Lowry protein assay, despite that the method measures plasma protamine levels whereas the Lowry assay measures protamine in aqueous samples. The slope and y-intercept, as estimated by the least-squares fitting with a correlation coefficient of 0.992 , are 0.940 and $1.9 \mu \mathrm{g} / \mathrm{mL}$, respectively, indicating that the proportional error of the method is $6.0 \%$ and the constant error is $1.9 \mu \mathrm{g} / \mathrm{mL}$. For a protamine concentration of $100 \mu \mathrm{g} / \mathrm{mL}$ by the Lowry assay, the dye method yields a mean value of 96.0 $\mu \mathrm{g} / \mathrm{mL}$, and the $95 \%$ confidence interval would be $93.5-98.5 \mu \mathrm{g} / \mathrm{mL}$.

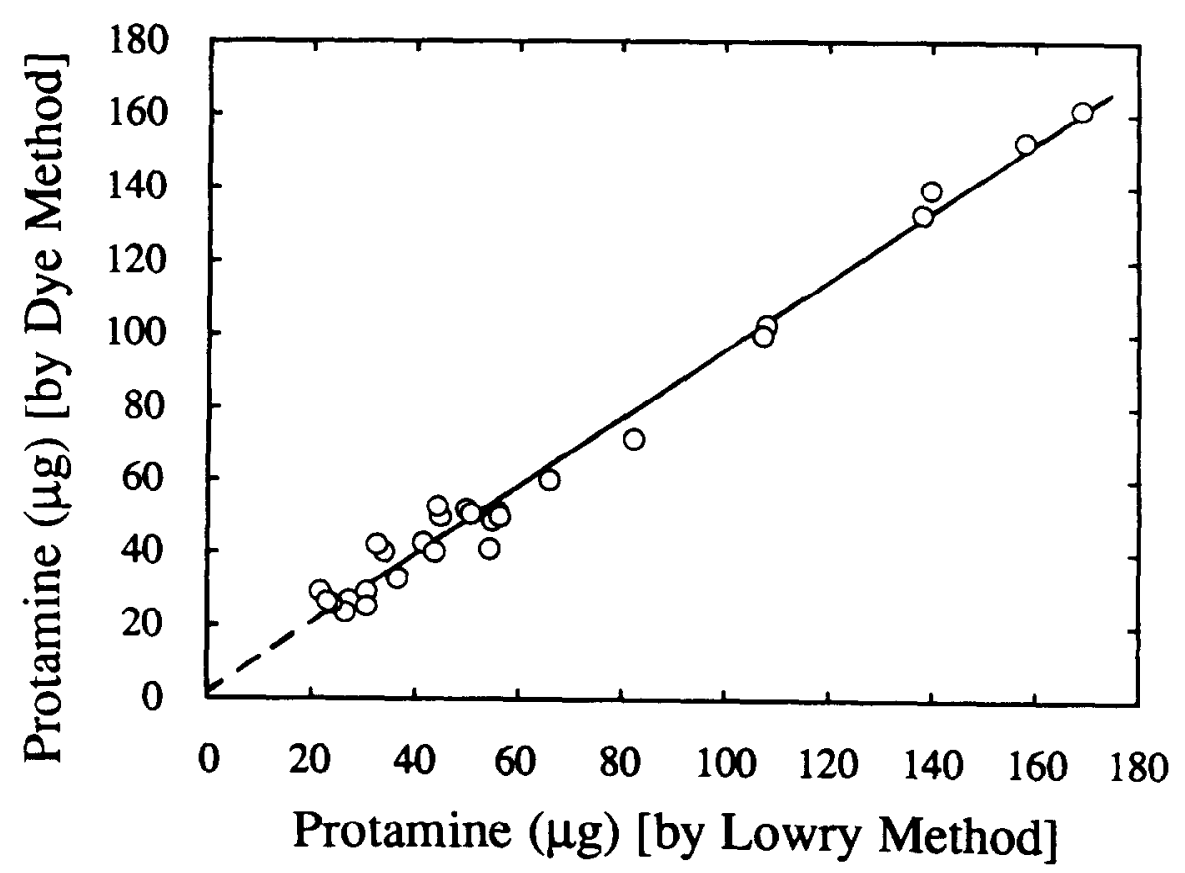

FIG. 2

Comparison of the plasma protamine concentrations determined by the azure A dye method with their aqueous counterparts determined by the conventional Lowry protein assay. To avoid overcrowding, only the average value of each data point was presented in the figure. The method comparison procedures described by Westgard and Hunt (8), which utilized the averaged values for linear regression, were followed for statistical analysis (see "Materials and Methods" for details). 


\section{TABLE I}

\section{Effect of Sample Variation on the Slope of the Standard Curve}

Sample Number

$\begin{array}{ll}1 & 0.0265 \pm 0.0044 \\ 2 & 0.0238 \pm 0.0012 \\ 3 & 0.0247 \pm 0.0005 \\ 4 & 0.0277 \pm 0.0001 \\ 5 & 0.0278 \pm 0.0006 \\ 6 & 0.0258 \pm 0.0010 \\ 7 & 0.0243 \pm 0.0005\end{array}$

* Each experiment was performed in triplicate.

In Table $I$ is a summary of the data of slopes of the 3-unit standard curves prepared on seven different plasma specimens. The slopes appear to be consistent over various plasma samples tested, with a mean value of 0.0258 and a standard deviation of 0.0016 .

In the heparinized plasma sample, the method apparently measures the remaining protamine level after heparin neutralization (i.e. the protamine excess), rather than the total protamine content in the sample. When $216 \mu \mathrm{g}$ of protamine were added to a sample containing 6 units of heparin, the protamine level determined by the method using a 3-unit standard curve was only $152.8 \mu \mathrm{g}$. Calculation from the difference in protamine content yielded a protamine/heparin neutralization ratio of $10.5 \mu \mathrm{g} / \mathrm{unit}$. This value is consistent with that of $10.3 \pm 0.5 \mu \mathrm{g} / \mathrm{unit}$ obtained from the protamine titration experiments using the procedures described previously (7) and the same batch of protamine and heparinized plasma. In addition, when 14.5 units of heparin (estimated from the protamine content of $152.8 \mu \mathrm{g}$ and using the above neutralization ratio of $10.5 \mu \mathrm{g} /$ unit) were added to back-titrate the protamine excess, the plasma aPTT was increased from the normal clotting time of $33.5 \pm 0.5$ seconds to $38.2 \pm 1.03$ seconds. Estimation made from a heparin-aPTT standard curve (prepared on plasma from the same donor) indicated that such an aPTT value (i.e. 38.2 seconds) was equivalent to a heparin level (due to over titration) of $0.5 \pm 0.1$ units. Based on this neutralization study, a protamine level of $147.2 \mu \mathrm{g}$ (as compared to the level of $152.8 \mu \mathrm{g}$ measured by the dye method) was calculated to be present in the "protamine excess" sample. These results all confirm the hypothesis that the method measures protamine excess.

\section{DISCUSSION}

Protamine is a strongly cationic polypeptide that is used regularly following cardiopulmonary bypass to neutralize the anticoagulant effect of heparin. Because an excess of protamine is necessary to maintain heparin inactivation, insufficient protamine may result in "heparin rebound", leading to postoperative bleeding (9). Conversely, too great an excess may cause adverse hemodynamic responses ranging from mild hypotension to severe, or ultimately fatal cardiovascular collapse (10). Inasmuch as protamine, itself, has anticoagulant properties $(11,12)$, a large excess may also enhance bleeding. Unfortunately, due to the lack of a specific method for the quantitation of protamine in plasma, the minimum and most effective protamine dose required to maintain neutralization of heparin has never been successfully determined.

Azure A dye is known to undergo a shift of its adsorption spectrum from blue to purple in the presence of heparin (6). Indeed, this unique metachromatic characteristic of azure $A$ dye has been used to measure the chemical quantities of heparin in plasma samples (13). Since the binding between azure $A$ and heparin is reversible and being relatively weak compared to that between protamine and heparin, when protamine is added to the heparin-azure A dye complex, it will displace azure A dye from the complex and subsequently restore the dye's original adsorption 
spectrum. Based on this competitive binding displacement mechanism, and also on the clinical specificity of protamine as heparin antagonist, we have developed a colorimetric method that is specific to measure plasma protamine levels. The plasma protamine levels determined by this dye method are within $94 \%$ of accuracy when compared with their aqueous counterparts determined by the conventional Lowry protein assay.

In the method presented, a plasma volume of $400 \mu \mathrm{L}$ and a total azure A dye content of $48 \mu \mathrm{g}$ were used for the method. Although theoretically the application of a larger sample volume and/or higher dye content would improve the detection limit of the method, they would also introduce a higher turbidity (due to plasma) and background absorbance (due to azure $A$ dye) to the sample. Based on our results (data are not shown), a plasma volume ranging from 300-400 $\mu \mathrm{L}$ and a dye content ranging from $40-50 \mu \mathrm{g}$ seem to be the optimum values.

Determination of an adequate standard curve for protamine quantitation is possibly the most difficult part for the method. In order for the method to function accurately, the linear region of the standard curve must fully cover the protamine concentrations to be measured. An approach is suggested herein which could facilitate the screening process for the most adequate standard curve. The approach calls for the addition of an excessive amount of heparin (e.g. 10 units) and protamine (e.g. $150 \mu \mathrm{g})$ to two separate test samples before mixing theses test samples $(400 \mu \mathrm{L})$ with an assay mixture $(600 \mu \mathrm{L})$ containing heparin $(5 \mathrm{units} / \mathrm{mL})$ and azure $A$ dye $(80 \mu \mathrm{g} / \mathrm{mL})$. The absorbance in these samples are measured at $620 \mathrm{~nm}$ and used as two extremes. To screen for the most adequate standard curve, $400 \mu \mathrm{L}$ of the test plasma samples are added to $600 \mu \mathrm{L}$ of the assay mixtures containing different standard heparin concentrations (i.e. ranging from 3.3 to 15 units $/ \mathrm{mL}$ as described in the METHOD). The heparin-containing assay mixture which provides a linear absorbance range that falls best within the aforementioned two absorbance extremes would be the one that yields the most adequate standard curve.

In theory, there should be no limits with regard to how much heparin can be used in the assay mixture. The lower/higher the protamine content, the lower/higher the heparin concentration is required in the assay mixture. In practice, however, final heparin concentrations of 2 and 9 units/mL are set as the lower and upper limits, respectively, for the method. Heparin concentrations below 2 units $/ \mathrm{mL}$ would produce an insufficient metachromatism, while above 9 units $/ \mathrm{mL}$ cause the formation of turbid heparin-protamine precipitates due to the presence of high levels of the compounds.

The method proves to measure the "free" protamine level after heparin neutralization (i.e. protamine excess), rather the total added dose of protamine. This behavior is somewhat anticipated, since only free protamine would have the ability to bind with heparin and thus replace azure A dye from the heparin-dye complexes. Because of this behavior, it is conceivable to employ the method during clinical reversal of heparin with protamine to monitor protamine excess. Studies in this direction are currently in progress, as are experiments aimed at gaining more insight into the effects of protamine excess, using clinical samples obtained from patients undergoing cardiopulmonary bypass who have received heparin and subsequent reversal with protamine. Preliminary results seem to confirm the method in measuring protamine excess, although the excess amount of protamine estimated by the method is considerably lower than that calculated by the administered heparin and protamine doses. Previous experiments involving dogs indicated that a significant amount of intravenous protamine disappeared during the in vivo studies, possibly due to its binding to endothelium cells (data are not shown).

The non-variability of the slope of the standard curve versus different plasma samples should provide an important and also beneficial feature towards both the ongoing clinical studies and the practical applicability of the method. In order for the method to measure protamine accurately, a standard curve must be prepared on plasma samples containing no heparin. Given the possibility that during an operational procedure the original plasma samples might not have been obtained prior to the heparinization of the patient, this invariable property would allow protamine levels to be determined using a standard curve prepared on a different plasma. 
The method may also assist in ascribing the clinical cause of the "heparin rebound" phenomenon (14). Shanberge and co-workers have suggested that the production of heparin rebound following heparin neutralization with protamine may be associated to the loss of protamine excess (9). Since the method measures protamine excess, it may help confirm such a hypothesis.

Lastly, because the method can precisely measure protamine in a rather complex medium (e.g. plasma), it may be used as a tool for the quality control during the preparation of protamine injectables, or for monitoring protamine during its isolation and purification process.

\section{ACKNOWLEDGMENTS}

This study was supported in part by the National Institute of Health Grant HL38353.

\section{REFERENCES}

1. ANDO T., YAMSAKI, M., and SUZUKI K. Protamines. In: Molecular Biology Biochemistry and Biophysics. A. Kleinzeller, G.F. Springer, and H.G. Wittmann (Eds.) Vol. 12. pp. 1-30, Berlin. Springer-Verlag (1973).

2. O'REILLY, R.A. Anticoagulant, antithrombotic, and thrombolytic drugs. In: The Pharmacological Basis of Therapeutics. A.G. Goodman, L.S. Gilman, and A. Gilman (Eds.) pp. 1347-1366, New York. Macmillan (1980).

3. JAQUES, L.B. Protamine-antagonist to heparin. J Can Med Assoc 108, 1291-1297, 1973.

4. LOWRY O.H., ROSEBROUGH, N.J., FARR, A.L., and RANDALL, R.J. Protein measurements with the Folin phenol reagent. J Biol Chem 193, 265-275, 1951.

5. BRADFORD, M.M. A rapid and sensitive method for the quantitation of microgram quantities of protein utilizing the principle of protein-dye binding. Anal Biochem 72, 248-254, 1976.

6. JAQUES, L.B., and WOLLIN, A. A modified method for the colorimetric determination of heparin. Can J Physiol Pharmacol 45, 787-794, 1967.

7. TENG, C.L.C., and YANG, V.C. A facile colorimetric protamine titration method. J Lab Clin Med 113, 498-504, 1989.

8. WESTGARD, J.O., and HUNT, M.R. Use and interpretation of common statistical tests in method-comparison studies. Clin Chem 19, 49-57, 1973.

9. SHANBERGE, J.N., MURATO, M., QUATTROCIOCCHI-LONGE, T., and VAN NESTE, L. Heparin-Protamine complexes in the production of heparin rebound and other complications of extracorporeal bypass procedures. Am J Clin Pathol 87, 210-217, 1987.

10. HORROW, J.C. Protamine: A review of its toxicity. Anesth Analg 64, 348-361, 1985.

11. PORTMANN, A.F., and HOLDEN, W.D. Protamine (salmine) sulfate, heparin and blood coagulation. J Clin Invest 28, 1451-1458, 1949.

12. SHANBERGE, J.N., BARLAS, A., and REGAN, E.E. The effect of protamine on thromboplastin generation. J Lab Clin Med 52, 744-753, 1958.

13. KLEIN, M.D., DRONGOWSKI, R.A., LINHARDT, R.J., and LANGER, R. A colorimetric assay for chemical heparin in plasma. Anal Biochem 124, 59-64, 1982.

14. BICK, R.L. Alterations associated with cardiopulmonary bypass: Pathophysiology, prevention, diagnosis and management. Sem Thromb Hemostas 3, 59-82, 1976. 